Discussion Paper No. 10-057

Wirkungen eines Betreuungsgeldes bei bedarfsgerechtem Ausbau frühkindlicher Kindertagesbetreuung: Eine Mikrosimulationsstudie

Denis Beninger, Holger Bonin, Julia Horstschräer, and Grit Mühler

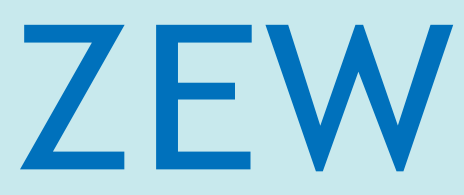

Zentrum für Europäische Wirtschaftsforschung $\mathrm{GmbH}$

Centre for European

Economic Research 
Discussion Paper No. 10-057

\section{Wirkungen eines Betreuungsgeldes bei bedarfsgerechtem Ausbau frühkindlicher Kindertagesbetreuung: Eine Mikrosimulationsstudie}

Denis Beninger, Holger Bonin, Julia Horstschräer, and Grit Mühler

Download this ZEW Discussion Paper from our ftp server:

ftp://ftp.zew.de/pub/zew-docs/dp/dp10057.pdf

Die Discussion Papers dienen einer möglichst schnellen Verbreitung von neueren Forschungsarbeiten des ZEW. Die Beiträge liegen in alleiniger Verantwortung der Autoren und stellen nicht notwendigerweise die Meinung des ZEW dar.

Discussion Papers are intended to make results of ZEW research promptly available to other economists in order to encourage discussion and suggestions for revisions. The authors are solely responsible for the contents which do not necessarily represent the opinion of the ZEW. 


\section{Das Wichtigste in Kürze}

Öffentliche Kindertagesbetreuung wird in Deutschland im internationalen Vergleich recht wenig genutzt. Es fehlt nach wie vor an ausreichenden Angeboten insbesondere im frühkindlichen Bereich. Um die Vereinbarkeit von Familie und Beruf zu fördern, werden derzeit zusätzliche Mittel für eine Ausweitung des Angebots an Kindertagesbetreuung bereitgestellt. Ziel ist, bis 2013 den Bedarf an öffentlicher Betreuung zu decken. Für diesen Zeitpunkt ist außerdem die Einführung eines Betreuungsgeldes geplant, wodurch Eltern, die keine öffentlich geförderte Kindertagesbetreuung in Anspruch nehmen, einen monetären Transfer für ihre eigene Erziehungsarbeit erhalten würden. Diese Maßnahme soll die Wahlfreiheit der Eltern zwischen eigener und externer Betreuung gewährleisten.

Unser Beitrag geht der Frage nach, welche Wirkungen von der Einführung eines Betreuungsgeldes in Deutschland zu erwarten sind. Dabei betrachten wir insbesondere die folgenden Größen: i) Arbeitsangebot, ii) Umfang der externen, öffentlich geförderten Kindertagesbetreuung, iii) Intensität der Kinderbetreuung innerhalb der Familie, iv) Haushaltseinkommen und v) fiskalische Kosten. Um die potenziellen Wirkungen bei gleichzeitigem Ausbau der öffentlich geförderten Kinderbetreuung ex ante abzuschätzen, nutzen wir basierend auf den Daten des Sozio-Ökonomischen Panels (SOEP) das Instrument der Mikrosimulation. Unser strukturelles Modell schätzt die Inanspruchnahme des Betreuungsgeldes und das Arbeitsangebot von Müttern und Vätern simultan und berücksichtigt eine mögliche Rationierung der Eltern hinsichtlich der Betreuungsmöglichkeiten für Kinder unter drei Jahren.

Gemäß unserer Simulation würden bei einem bedarfsgerechten Ausbau der Kindertagesbetreuung die Inanspruchnahme und das Arbeitsangebot moderat wachsen. Das Betreuungsgeld hingegen wirkt entgegengesetzt. Es führt zu einer moderaten Abnahme des Arbeitsangebots der Mütter und der Nachfrage nach externer Kinderbetreuung. Eine gleichzeitige Betrachtung dieser beiden Entwicklungen resultiert in einem insgesamt fallenden Arbeitsangebot, wobei 5,1\% der Mütter ihr Arbeitsangebot ausweiten und 8,1\% weniger aktiv auf dem Arbeitsmarkt wären. Auch in Bezug auf die Inanspruchnahme von öffentlicher Betreuung überwiegt der negative Effekt des Betreuungsgeldes. Die Inanspruchnahme von öffentlicher Betreuung sinkt um 4,1 Prozentpunkte. Zwar verbessert sich mit dem Betreuungsgeld die Einkommensposition bildungsferner Familien, gleichzeitig stärkt es aber in diesen Familien auch die bereits bestehende Neigung Kleinkinder zu Hause zu betreuen. 


\section{Non-Technical Summary}

Publicly funded day care for children under the age of 3 is used less frequently in Germany compared to other (especially Nordic) countries. Supply of day care is still scarce for this age group. In order to improve possibilities to combine family duties with employment, additional public resources are currently provided for the expansion of publicly funded day care. The aim is to meet demand by 2013. For that year it is also planned to introduce a home care allowance, which is basically a monetary transfer for parents that do not use publicly funded day care. The idea is to subsidize child care within families and external care similarly, so that parents can decide for their preferred child care arrangement without any monetary considerations.

Our paper analyzes the impact of the introduction of a home care allowance for children between 13 and 36 months of age and explicitly takes the expansion of publicly funded day care into account. We especially investigate the impact on i) labor supply, ii) public day care usage, iii) intensity of parental care, iv) household income and v) fiscal costs. We use the German Socio-Economic Panel (GSOEP) to estimate a structural model in which parents decide simultaneously about labor supply, usage of external day care and their time spent for child care within the family. The model also allows for possible rationing of parents with respect to publicly funded care arrangements.

Simulations using the estimated model parameters show that labor supply and day care usage would increase moderately once publicly funded day care provision meets demand. The introduction of a home care allowance instead would lead to a moderate reduction in mother's labor supply as well as in day care usage. Considering both reforms at the same time, we overall find a decreasing labor supply, with $5.1 \%$ of the mothers increasing their labor market activities and $8.1 \%$ reducing it. The negative effects of the home care allowance also outweigh the impact of the expanding day care provision with respect to day care usage. Attendance rates decline by 4.1 percentage points. A home care allowance in fact increases the household income especially for poorly educated families, but at the same time discourages these parents to use external day care which can be important for early education. 


\title{
Wirkungen eines Betreuungsgeldes bei bedarfsgerechtem Ausbau frühkindlicher Kindertagesbetreuung: Eine Mikrosimulationsstudie*
}

\author{
Denis BENINGER \\ (HWR Berlin und Université de Strasbourg) \\ Holger BONIN \\ (ZEW Mannheim und IZA Bonn) \\ Julia HORSTSCHRÄER \\ (ZEW Mannheim) \\ Grit MÜHLER \\ (ZEW Mannheim)
}

\begin{abstract}
Zusammenfassung
Der Beitrag evaluiert die Wirkungen eines Betreuungsgeldes bei gleichzeitigem Ausbau der öffentlich geförderten Tagesbetreuung für Kinder im Alter von 13 bis 36 Monaten. Wir schätzen mit SOEP-Daten und unter Berücksichtigung partiell beobachtbarer Rationierungen im Betreuungsbereich ein strukturelles Modell, in dem Eltern simultan über den Umfang des Arbeitsangebots, den Umfang externer Betreuung und die Intensität der Kinderbetreuung in der Familie entscheiden. Simulationen auf Grundlage der geschätzten Modellparameter ergeben, dass ein Betreuungsgeld das Arbeitsangebot und die Nachfrage nach externen Betreuungsangeboten spürbar verringert. Für sich betrachtet wirkt der Ausbau der Kindertagesbetreuung bei beiden Zielgrößen in die umgekehrte Richtung. Im Gesamteffekt beider Maßnahmen fallen sowohl das Arbeitsangebot als auch die Inanspruchnahme von Betreuung außerhalb der Familie. Zwar verbessert sich die Einkommensposition bildungsferner Familien, ein Betreuungsgeld behindert bei dieser Gruppe aber die Nutzung externer frühkindlicher Bildungsangebote.
\end{abstract}

JEL Klassifikation:J13, D13, H23

Keywords: Behavioral Microsimulation, Day Care, Family Policy, Germany

\footnotetext{
* Der Beitrag enthält Ergebnisse einer im Auftrag des Bundesministeriums der Finanzen erstellten Studie „Fiskalische Auswirkungen sowie arbeitsmarkt- und verteilungspolitische Effekte einer Einführung eines Betreuungsgeldes für Kinder unter 3 Jahren.“ Der Inhalt liegt in alleiniger Verantwortung der Autoren und stellt nicht notwendigerweise die Meinung des Auftraggebers der Studie oder des ZEW dar. Die Autoren bedanken sich bei Markus Clauss für wertvolle Forschungsunterstützung. Korrespondierender Autor: Holger Bonin, Zentrum für Europäische Wirtschaftsforschung, L7 1, 68161 Mannheim, bonin@zew.de.
} 


\section{Einführung}

Bei der Bereitstellung von öffentlich geförderter Kindertagesbetreuung liegt Deutschland im internationalen Vergleich deutlich zurück. Nur jedes sechste Kind unter drei Jahren wird in einer Kindertageseinrichtung betreut; vielfach fehlt es nach wie vor an ausreichenden Angeboten (BMFSFJ 2009). Allerdings haben sich die Akzente der deutschen Familienpolitik zuletzt verschoben. Um die Vereinbarkeit von Beruf und Familie zu fördern und Anreize zur Familiengründung zu geben, sollen in den nächsten Jahren mehr öffentliche Mittel in den Ausbau der Betreuung für Kleinkinder fließen. Jedoch ist diese Politik durchaus umstritten. Kritiker argumentieren, dass der Wunsch, Kinder selbst zu erziehen, genauso staatlich gefördert werden solle, um den Eltern Wahlfreiheit zu belassen und eigene Erziehungsarbeit stärker anzuerkennen. Dies hat zu Vorschlägen für ein Betreuungsgeld geführt. Hierbei handelt es sich um einen monetären Transfer an Eltern, die keine öffentlich geförderte Kindertagesbetreuung in Anspruch nehmen. Mit der Einführung dieser familienpolitischen Leistung würde Deutschland Vorbildern in einigen skandinavischen Ländern folgen. In Deutschland existiert ein Betreuungsgeld bisher nur auf der Länderebene in Thüringen.

Dieser Beitrag geht der Frage nach, welche Wirkungen von der Einführung eines Betreuungsgeldes in Deutschland zu erwarten sind. Zu rechnen ist insbesondere mit Veränderungen beim Arbeitsangebot und bei der Wahl der Kinderbetreuung durch Familien mit kleinen Kindern. Für Norwegen und Finnland liegen einige empirische Erkenntnisse zu kausalen Effekten von Betreuungsgeldprogrammen vor, die sich wegen der andersartigen familienpolitischen Systeme aber nur bedingt übertragen lassen. Dagegen fehlt es bisher an geeigneten Daten für eine Evaluation des Thüringer Programms. Darüber hinaus muss eine Bewertung eines bundesweiten Betreuungsgeldes die regional unterschiedliche Versorgung mit Kinderbetreuungsplätzen, ebenso wie den noch stattfindenden Ausbau des Betreuungsangebots vor allem in den westdeutschen Bundesländern, mit in den Blick nehmen. Daher wählen wir für diese Studie den Weg der ex ante-Evaluation mithilfe einer verhaltensbasierten Mikrosimulation auf Grundlage von Daten des Sozio-Ökonomischen Panels (SOEP).

Das eingesetzte Mikrosimulationsmodell modelliert die Inanspruchnahme des Betreuungsgeldes und das Arbeitsangebot von Müttern und Vätern simultan und berücksichtigt darüber hinaus, dass Eltern hinsichtlich der Betreuungsmöglichkeiten für Kinder unter drei Jahren möglicherweise rationiert sind, also Art und Umfang der gewünschten Betreuung wegen eines fehlenden Angebotes möglicherweise nicht realisieren können. Eine solche Modellerweiterung 
ist im deutschen Kontext besonders erforderlich, da der Zusammenhang zwischen der Erwerbstätigkeit der Mütter und der Inanspruchnahme externer Kinderbetreuung nicht eindeutig ist. Zum einen nutzen viele Familien, in denen die Mutter nicht erwerbstätig ist, externe Kinderbetreuung. Zum anderen nehmen viele Familien, in denen beide Elternteile erwerbstätig sind, keine formelle Kinderbetreuung durch Kindertagesstätten oder Kindertagespflege in Anspruch, sondern nutzen kostengünstigere informelle Betreuungsarrangements (Wrohlich 2007).

Unser Modell erlaubt eine ex ante Abschätzung der Wirkungen eines Betreuungsgeldes hinsichtlich folgender Größen: i) Arbeitsangebot, ii) Umfang der externen, öffentlich geförderten Kindertagesbetreuung, iii) Intensität der Kinderbetreuung innerhalb der Familie, iv) Haushaltseinkommen und v) fiskalische Kosten. Da die Verhaltenswirkungen der Maßnahme für jeden Haushalt in der Mikrodatenbasis einzeln simuliert werden, erhalten wir zudem Wirkungsaussagen für verschiedene Haushaltstypen je nach persönlichen Merkmalen der Mütter oder der Einkommensposition vor der Reform.

Der weitere Artikel gliedert sich wie folgt: Abschnitt 2 verschafft einen Überblick über die Erfahrungen mit bestehenden Betreuungsgeldmaßnahmen. Abschnitt 3 beschreibt das eingesetzte Mikrosimulationsmodell. Abschnitt 4 enthält die Ergebnisse der ex ante Wirkungsanalyse. Abschnitt 5 zieht die Schlussfolgerungen.

\section{Erfahrungen mit einem Betreuungsgeld}

\subsection{Finnland und Norwegen}

Der Vorreiter bei der Einführung eines Betreuungsgeldes ist Finnland, das seit 1985 eine monatliche „Child Home Care Allowance“ an Eltern auszahlt, die ihre Kinder selbst oder von einer Tagespflegeperson im eigenen Haushalt betreuen lassen. Mit Auslaufen des Elterngeldes ab dem neunten Lebensmonat des Kindes setzt die Zahlung des Betreuungsgeldes ein, das bis zum 36. Lebensmonat des Kindes bezogen werden kann. Dieses Betreuungsgeld besteht aus einer Grundpauschale (1994: 329 Euro; 1996: 252 Euro; 2010: 314 Euro) und einem einkommensabhängigen Teil, der sich reduziert, sobald das Haushaltseinkommen einen bestimmten Betrag überschreitet. Das durchschnittlich erhaltene Betreuungsgeld betrug 1985 zunächst 163 Euro pro Haushalt und erhöhte sich bis 1996 auf 333 Euro. Derzeit beträgt der Höchstbetrag - inklusive einkommensabhängigen Zuschlag - 482 Euro. 
Die Einführung eines Betreuungsgeldes in Norwegen folgte im August 1998 mit der sogenannten „Cash for Care Subsidy“. Zu Beginn waren nur die Eltern von 13-24 Monate alten Kindern anspruchsberechtigt, ab Januar 1999 waren es dann alle Eltern mit Kindern im Alter von 13 bis 36 Monaten. Die Höhe des gezahlten Betrags orientiert sich an den monatlichen Kosten für die Inanspruchnahme einer Kinderbetreuungseinrichtung. Sie stieg von anfänglich ca. 370 Euro auf ca. 450 Euro an, die seit dem Jahr 2003 an norwegische Eltern gezahlt werden, die ihre Kinder zu Hause betreuen (lassen). ${ }^{1}$

Sowohl in Norwegen als auch in Finnland folgt das Betreuungsgeld auf das Elterngeld, das nur im ersten Lebensjahr des Kindes gezahlt wird. Es bestehen jedoch geringfügige Unterschiede in der Ausgestaltung des Betreuungsgeldes zwischen den beiden Ländern. In Finnland, nicht aber in Norwegen, müssen die Eltern den erhaltenen Betrag versteuern. Dafür können finnische Eltern einen Geschwisterzuschlag in Höhe von 20\% der Grundpauschale zusätzlich erhalten. Eine anteilige Auszahlung des Betreuungsgeldes bei einem TeilzeitKrippenbesuch erfolgt wiederum nur in Norwegen, während der Anspruch in Finnland entfällt, sobald die Kinder eine öffentlich geförderte Einrichtung besuchen.

Die Quoten der Betreuungsgeld beziehenden Eltern in Relation zu allen Eltern mit Kindern im entsprechenden Alter zeigen eine hohe Inanspruchnahme. In Norwegen nehmen ca. 80\% der Eltern und mehrheitlich Mütter das Betreuungsgeld in Anspruch, nur 5\% der Empfänger sind Väter (Schøne 2004). Finnische Daten von 1996 zeigen, dass nur in 15\% der Fälle die Eltern selbst ihr Kind betreuen, die große Mehrheit der Eltern jedoch das Betreuungsgeld zur Anstellung einer Tagespflegeperson nutzt (Sipilä/Korpinen 1998).

Aussagen zu durch das Betreuungsgeld ausgelösten Änderungen im Betreuungsverhalten gestalten sich schwierig. In Norwegen ging die Einführung mit dem Ausbau der qualitativ hochwertigen, öffentlich geförderten Kindertagesbetreuung einher. So stieg zwischen 2004 und 2007 die Quote der betreuten Einjährigen von 37,8\% auf 61,4\%, die der betreuten Zweijährigen von 57,8\% auf 79,5\% (Statistics Norway 2003, 2007). Empirische Studien, die das norwegische Programm „Cash For Childcare“ untersuchen (Hardoy/Schøne 2008, Naz 2004, Schøne 2004), können jedoch zeigen, dass von der Zahlung des Betreuungsgeldes negative Wirkungen auf das Arbeitsangebot norwegischer Frauen ausgehen. In der Zeit, in der sie anspruchsberechtigt sind, liegt die Erwerbsbeteiligung von Frauen um vier Prozentpunkte nied-

\footnotetext{
${ }^{1}$ Gleichzeitig wurde allerdings eine zusätzliche Förderung von Kindern unter 3 Jahren abgeschafft, so dass mit der Erhöhung des Betreuungsgeldes keine Entlastung der Familien einherging.
} 
riger. Das Arbeitsangebot von Frauen mit Migrationshintergrund (aus nicht-westlichen Ländern) sinkt sogar um fünf bis zehn Prozentpunkte. Die negativen Partizipationseffekte wachsen mit dem Bildungsgrad der Mütter.

Der Rückgang der Erwerbsbeteiligung ist etwas höher als der der Arbeitszeit (Schøne 2004). Bei anspruchsberechtigten Frauen sinkt die Jahresarbeitszeit um etwa 3\%. Negative Lohneffekte bei Wiedereintritt in den Arbeitsmarkt sind allerdings nicht zu beobachten, da die Dauer der Erwerbsunterbrechung mit 3-5 Monaten im Durchschnitt eher gering ist und viele Eltern auch im ersten Lebensjahr bereits häufiger Tagesmütter in Anspruch nehmen. Bei der Einordnung dieser Ergebnisse ist auch zu berücksichtigen, dass Norwegen allgemein eine höhere Müttererwerbsquote als Deutschland aufweist. Dies gilt auch nach dem mit der Einführung des Betreuungsgeldes verbundenen Rückgang.

Empirische Studien für Finnland hingegen zeigen auch bei der Inanspruchnahme öffentlicher Betreuung die erwartbaren Wirkungen: die Betreuung durch öffentliche Kindertageseinrichtungen ging zurück (Ilmakunnas 1997). Gleichzeitig verringerten auch in Finnland Frauen mit Kindern unter drei Jahren ihr Arbeitsangebot. 1993 arbeiteten bereits nur 56\% dieser Frauen, während 1995 nur noch 48\% (Sipilä/Korpinen 1998) erwerbstätig waren.

Eine kausale Schätzung des Wirkungszusammenhangs gestaltet sich jedoch schwierig. Während Schøne (2004) mit Hilfe eines regressionsadjustierten Differenz-von-DifferenzenSchätzers auf die Schätzung eines kausalen Wirkungszusammenhangs für die norwegische Reform abzielt (jedoch nicht für zeitveränderliche Faktoren kontrollieren kann), könnte der von Sipilä/Korpinen (1998) für Finnland beobachtete Rückgang auch durch sich verschlechternde Arbeitsmarktbedingungen beeinflusst worden sein. Die Evidenz für einen negativen Zusammenhang zwischen dem Betreuungsgeld und dem Arbeitsangebot von Frauen ist daher für Finnland etwas schwächer.

\section{2 „Thüringer Erziehungsgeld“}

Im Rahmen der „Thüringer Familienoffensive“ trat zum 1. Juli 2006 ein einkommensunabhängiges Betreuungsgeld für Kinder im dritten Lebensjahr, das sogenannte „Thüringer Erziehungsgeld“, an die Stelle des zuvor gezahlten, einkommensabhängigen Landeserziehungsgeldes (Opielka/Winkler 2009). Erklärtes Ziel der Landesregierung war es, die Wahlfreiheit der Eltern zu stärken. Das Land zahlt seitdem für die Dauer eines Jahres monatlich 150 Euro für das erste Kind, 200 Euro für das zweite, 250 Euro für das dritte und 300 Euro für jedes weite- 
re Kind zwischen 24 und 36 Monaten. Beim Besuch einer Kindertagesstätte (oder bei Betreuung durch eine Tagespflegeperson) fließen je nach zeitlicher Inanspruchnahme bis zu 100\% des Erziehungsgeldes direkt in die Finanzierung des Betreuungsplatzes. Damit wird gleichzeitig die gewünschte stärker nachfrageorientierte Finanzierung erreicht. Mit den verfügbaren Daten zur Inanspruchnahme von Kindertageseinrichtungen aus der Kinder- und Jugendhilfestatistik von 2006-2008² lassen sich bisher einige grundlegende Zusammenhänge zwischen der Einführung des Betreuungsgeldes und dem Besuch einer Tageseinrichtung beschreiben. Es zeigen sich kleinere, nicht eindeutige Veränderungen bei der Anzahl der betreuten Zweijährigen und deutlich stärkere Verschiebungen im täglichen Betreuungsumfang.

Die Anzahl der in öffentlich geförderten Einrichtungen betreuten Zweijährigen ist im gesamten Zeitraum um 5,7\% zurückgegangen, die Betreuungsquote in öffentlich geförderter Kindertagespflege hingegen um 11,2\% gestiegen. In beiden Betreuungsformen hat der Anteil der betreuten zweijährigen Kinder von 2006 auf 2007 abgenommen, ist aber im Jahr darauf wieder gestiegen. ${ }^{3}$ Beim Umfang der Betreuung (in Stunden) zeigt sich in allen Altersgruppen eine Zunahme der täglichen Betreuungszeit in der Einrichtung. Diese Verschiebung ist besonders auffällig bei den Zweijährigen, bei denen im Jahr 2007 ein Viertel weniger Kinder für eine Zeitdauer von 5 Stunden täglich betreut werden, dafür jedoch 30\% mehr für eine Dauer zwischen 5 und 7 Stunden verglichen mit dem Vorjahr. Bei Familien mit Migrationshintergrund (gemessen an der in der Familie gesprochenen Sprache) oder besonderem Förderbedarf zeigen sich kaum Veränderungen. Es kann also nicht unmittelbar geschlussfolgert werden, dass die Leistung zu einer geringeren Inanspruchnahme öffentlich geförderter Kindertageseinrichtungen durch diese Gruppen geführt hat.

Zur Identifikation eines kausalen Zusammenhangs, zu dem sich die durch isolierte Einführung auf Länderebene gegebene quasi-experimentelle Situation grundsätzlich eignen würde, reichen die bisher verfügbaren Daten leider nicht aus. Die beobachteten Veränderungen in der Inanspruchnahme können mit der Einführung des Betreuungsgeldes zusammenhängen, müssen aber nicht ursächlich darauf zurückgeführt werden. Beim Stundenumfang zum Beispiel könnte das Betreuungsgeld dazu geführt haben, dass Eltern nun stärker die mit der (entgangenen) Zahlung zur Verfügung stehende Betreuungsdauer in der Einrichtung ausnutzen. Da je-

\footnotetext{
${ }^{2}$ Siehe Kolvenbach/Taubmann (2006) für eine ausführliche Darstellung der Daten.

${ }^{3}$ Die Veränderungen in der Betreuungsquote der Zweijährigen in Kindertageseinrichtungen sind für das Jahr 2006 auf $2007-5,8 \%$ und für das Jahr darauf +0,1\%. Die entsprechenden Veränderungen bei der Kindertagespflege betragen $-13,5 \%$ und $+24,7 \%$. Da die Anzahl der Kinder, die in öffentlich geförderter Kindertagespflege betreut werden, jedoch sehr gering ist, wirken sich Schwankungen hier prozentual besonders stark aus.
} 
doch gleichzeitig mit der Einführung des „Thüringer Erziehungsgeldes“ auch der Rechtsanspruch auf öffentlich geförderte Betreuung für Kinder ab zwei Jahren im Gesetz verankert wurde, könnte auch diese Maßnahme auf die beobachtete Ausdehnung der Betreuungszeit wirken. Für eine fundierte Evaluationsstudie wären genauere Daten erforderlich. Solange diese fehlen, können die möglichen Wirkungen der Einführung eines allgemeinen Betreuungsgeldes in Deutschland nur mithilfe der Methoden einer Ex ante-Evaluation per Mikrosimulation abgeschätzt werden. Der folgende Abschnitt beschreibt ein dafür geeignetes verhaltensbasiertes strukturelles empirisches Modell.

\section{Mikrosimulationsmodell zur Ex ante-Evaluation}

Zur ex ante-Bewertung eines Betreuungsgeldes nutzen wir ein verhaltensgestütztes Mikrosimulationsmodell. Damit lassen sich die Wirkungen von Veränderungen im Steuer- und Transfersystem unter der Annahme unveränderter Verhaltensparameter, die die Präferenzen der Haushalte repräsentieren, im Vergleich zum Status quo simulieren. Das eingesetzte Modell geht über das auf dem Feld der Mikrosimulation verbreitete diskrete Arbeitsangebotsmodell hinaus, weil sowohl die externe Kinderbetreuungszeit als auch die Betreuungszeit der Mütter als endogen betrachtet wird. ${ }^{4}$ Dies ist erforderlich, da der Leistungsumfang beim Betreuungsgeld unmittelbar an das gewählte Betreuungsarrangement geknüpft ist.

\subsection{Strukturelles Modell der Wahl von Arbeitsangebot und Kinderbetreuung}

Ausgangspunkt unseres Modells ist das neoklassische Modell des Arbeitsangebots. Dieses Modell bildet die Handlungsoptionen eines Haushalts mit Hilfe einer Nutzenfunktion ab, die positiv von zwei Argumenten abhängt: der verfügbaren Freizeit, also der Zeitausstattung abzüglich der geleisteten Arbeit am Markt, und dem verfügbaren Einkommen. Das verfügbare Einkommen ergibt sich aus dem bei der gewählten Arbeitszeit erzielten Bruttoeinkommen unter Berücksichtigung der vom Haushalt zu leistenden Steuern und empfangenen Transfers. Da sich die Konsummöglichkeiten durch Aufgabe von Freizeit zur Erzielung von Einkommen steigern lassen, stehen die Haushaltsmitglieder vor einem Entscheidungsproblem: Sie müssen

\footnotetext{
${ }^{4}$ Für Mikrosimulationsmodelle mit diskreter Entscheidung über das Arbeitsangebot im Haushaltskontext vgl. etwa Bonin et al. 2003, Steiner/Wrohlich 2005, Arntz et al. 2007. Anwendungen zur Evaluation familienpolitischer Reformen finden sich im Bereich der Besteuerung (etwa Beblo et al. 2004, Fuest et al. 2006, Maiterth 2004, Steiner/Wrohlich 2004, 2008), des Transfersystems (etwa Dearing et al. 2007, Spieß/Wrohlich 2008) und der Kinderbetreuungskosten (etwa Beblo et al. 2005 und Wrohlich 2007).
} 
zwischen dem Nutzenzuwachs durch mehr Konsum bei mehr Arbeit und dem Nutzenverlust durch weniger Freizeit abwägen.

Das übliche Arbeitsangebotsmodell wird für den vorliegenden Zweck um ein Modell der Wahl der Kinderbetreuung erweitert. Unser Modell unterstellt, dass Mütter simultan über ihre Arbeits- und Kinderbetreuungszeit entscheiden. Die zur Kinderbetreuung eingesetzte Zeit gilt für sie nicht länger als Freizeit, sondern wird als direktes Argument in die Nutzenfunktion aufgenommen. Es existiert also neben der Freizeitpräferenz eine Präferenz für Kinderbetreuungszeit. Im ökonometrischen Modell korrigiert diese Spezifikation eine mögliche systematische Überschätzung der Freizeitpräferenz von Müttern, die in den Daten signifikant niedrigere Arbeitszeiten aufweisen als Männer.

Das Entscheidungsmodell wird darüber hinaus dahingehend erweitert, dass Eltern ihre Kinder nicht nur selber betreuen, sondern auch Betreuung in öffentlichen oder privaten Betreuungseinrichtungen in Anspruch nehmen können. Eine externe Kinderbetreuung, die sowohl erwerbstätigen als auch nicht erwerbstätigen Eltern offen steht, verschafft Eltern zum einen mehr Freizeit. Sie geht allerdings zu Lasten anderen Konsums, denn je nach Art und Umfang fallen Kosten an. Diese hängen davon ab, ob Familien rationiert sind, also keinen Zugang zu öffentlich geförderter Kinderbetreuung haben und daher ggf. höhere Ausgaben für nicht subventionierte private Angebote tätigen müssen. Zum anderen kann die Inanspruchnahme externer Betreuungsarrangements einen direkten Nutzen stiften. Sie hat demnach einen eigenen Wert, etwa wegen wahrgenommenen Qualitätsunterschieden zwischen externer und eigener Betreuung. Im so erweiterten Modell stehen die Haushaltsmitglieder vor einem zweiten Entscheidungsproblem: Sie müssen zwischen dem möglichen Nutzenverlust durch weniger Konsum bei mehr externer Kinderbetreuung und den möglichen Nutzengewinnen aus mehr persönlicher Freizeit und externer Betreuung abwägen.

Um das skizzierte Modell für Simulationszwecke nutzen zu können, muss es in eine strukturelle Form überführt und empirisch geschätzt werden. Wir unterstellen erstens ein unitäres Entscheidungsmodell: Eltern poolen ihr Einkommen und maximieren eine gemeinsame Nutzenfunktion, die von der Freizeit von Müttern und Vätern, der Zeit für die Kinderbetreuung der Mutter, der Art der externen Kinderbetreuung und dem möglichen sonstigen Konsum abhängt. Zweitens basiert die ökonometrische Spezifikation auf einem diskreten Auswahlmodell in der Tradition von van Soest (1995). Dieses vereinfacht das Schätzproblem, indem dem Haushalt nur eine begrenzte Zahl von Handlungsmöglichkeiten gegeben wird. 
Konkret beschränken wir die Handlungsmöglichkeiten der Haushalte wie folgt: (1) Hinsichtlich der Arbeitszeit am Markt haben Frauen die Möglichkeiten der Nichtteilnahme, der Teilzeitarbeit oder der Vollzeitarbeit, Männer die Möglichkeiten der Nichtteilnahme und der Vollzeitarbeit. (2) Hinsichtlich der externen Kinderbetreuung gibt es drei Möglichkeiten: keine externe Betreuung, Halbtags- oder Ganztagsbetreuung. (3) Hinsichtlich der eigenen Kinderbetreuungszeit haben Mütter die Möglichkeiten intensiver Betreuung (35 Wochenstunden) und normaler Betreuung (16 Stunden). ${ }^{5}$ Für Väter ist die Kinderbetreuungszeit einheitlich auf 10,5 Stunden pro Woche festgesetzt. Somit haben die Haushalte im Modell $2 \times 3 \times 3 \times 2=36$ Handlungsmöglichkeiten.

Wie zur Identifikation diskreter Wahlmodelle üblich, nehmen wir an, dass die beobachtete Entscheidung der Haushalte unter den zugelassenen Alternativen die Alternative mit dem höchsten Wert der empirischen Nutzenfunktion repräsentiert. ${ }^{6}$ Für die Schätzung muss zudem die funktionale Form der Nutzenfunktion spezifiziert werden. Wir lassen zu, dass das Gewicht der in die Nutzenfunktion eingehenden Arbeitszeitvariablen in Abhängigkeit von persönlichen und Haushaltsmerkmalen, wie etwa dem Bildungsstand, dem Alter oder der Anzahl und Altersstruktur der Kinder im Haushalt, systematisch variiert. Schließlich treffen wir die Annahme, dass die nicht beobachteten Argumente der Nutzenfunktion über alle Alternativen des Haushalts unabhängig und identisch einer Extremwert-Verteilung folgen. Dies führt zu einem leicht schätzbaren konditionalen Logit-Modell (McFadden 1974).

\subsection{Empirische Spezifikation}

Die Parameter des strukturellen Modells zur Mikrosimulation werden auf Grundlage des SOEP geschätzt. Dieser Datensatz beinhaltet neben detaillierten sozio-demographischen Merkmalen der Haushalte auch geeignete Informationen über die zu erklärenden Verhaltensvariablen des Modells: Arbeitszeit, Kinderbetreuungszeit und Form der Kinderbetreuung. ${ }^{7} \mathrm{Da}$ die zu analysierende Politik nur eine vergleichsweise geringe Anzahl von Haushalten - Familien mit Kindern im Alter zwischen 13 und 36 Monaten - betrifft, poolen wir die Beobachtungen der in den Jahren 2002-2006 erfassten Haushalte, um ausreichende Fallzahlen zu erhalten.

\footnotetext{
${ }^{5}$ Diese Werte entsprechen etwa dem 33. bzw. 67. Perzentil der Verteilung der Kinderbetreuungszeiten bei deutschen Müttern gemäß der Zeitverwendungsstudie (Beblo 2001). Um eine Identifikation zu gewährleisten, geht die Zeit für Kinderbetreuung nicht linear, sondern als Indikatorvariable in das Schätzmodell ein.
} 
Für die Schätzung des diskreten Wahlmodells muss für jeden Haushalt das mit jeder Handlungsalternative - charakterisiert durch eine Arbeitszeit und ggf. externe Kinderbetreuung verbundene verfügbare Einkommen berechnet werden. Zur Ermittlung der Bruttoarbeitseinkommen wird zunächst für jedes Individuum ein Stundenlohn auf Basis der beobachteten Bruttolöhne und Arbeitszeiten der Beschäftigten unter Korrektur von Selektionsverzerrungen durch das Heckman-Verfahren geschätzt und mit den möglichen Arbeitszeiten kombiniert. Davon ausgehend wird das zugehörige verfügbare Haushaltseinkommen mit Hilfe eines Steuer-Transfer-Modells errechnet. Dieses Modell bildet das deutsche Steuer- und Transfersystem unter Berücksichtigung des laufenden Rechtsstands für jedes einzelne Jahr und unter Berücksichtigung individueller Haushaltsmerkmale äußerst detailliert nach (Clauss/Schubert 2009).

Vom so ermittelten verfügbaren Haushaltseinkommen sind ggf. externe Betreuungskosten je nach betrachteter Alternative abzuziehen. Bei öffentlich geförderter Kinderbetreuung können die Elternbeiträge, getrennt für Halbtags- und Ganztagsbetreuung, durch ein Tobit-Modell geschätzt werden. Gemäß den Schätzergebnissen ist ein niedrigeres Haushaltsnettoeinkommen mit signifikant niedrigeren Gebühren verbunden. Niedrigere Kosten fallen auch für Eltern an, wenn mindestens ein weiteres Kind eine Kindertageseinrichtung besucht. Darüber hinaus zeigen sich signifikante Unterschiede in Abhängigkeit vom Wohnort. ${ }^{8}$ Da das Angebot an öffentlich geförderter Kinderbetreuung begrenzt ist, unterstellen wir weiterhin, dass ein Anteil $p$ der Familien mit Kindern bei der Kinderbetreuung rationiert ist und eine private Kinderbetreuung in Anspruch nehmen müsste. Wie Wrohlich (2007) treffen wir die Annahme, dass der Preis auf dem privaten Betreuungsmarkt mit 5 Euro pro Stunde deutlich höher ist als bei Betreuung in einer öffentlichen, subventionierten Einrichtung.

Um die erwarteten Kinderbetreuungskosten vollständig zu schätzen, muss der Anteil $p$ der rationierten Familien ermittelt werden. Hiefür wird ein „Partial Observability Model“ genutzt, mit dem sich Nachfrage und Angebot eines rationierten Gutes simultan schätzen lassen (Poirier 1980, Abowd/Farber 1982 und Chevalier/Viitanen 2004). Das Modell wird auf Basis der SOEP-Welle 2002 geschätzt, da die benötigten Daten nur für dieses Jahr zur Verfügung stehen. Die Information, ob Eltern hinsichtlich der öffentlich geförderten Betreuung eines Kindes rationiert sind, muss aus den Daten konstruiert werden. Wir verwenden folgende Definiti-

\footnotetext{
${ }^{6}$ Hierbei kann sich ein Rundungsproblem ergeben, weil einige Individuen andere als die standardisierten Arbeitszeiten anbieten. Dieser Rundungsfehler ist erfahrungsgemäß praktisch aber eher unbedeutend.

${ }^{7}$ Vgl. Wagner et al. (2006) für eine genaue Beschreibung des SOEP.

${ }^{8}$ Eine Dokumentation der Ergebnisse dieser und aller weiteren Regressionen, die dem Mikrosimulationsmodell zugrunde liegen, ist bei den Verfassern auf Anfrage erhältlich.
} 
on: Eine Rationierung liegt bei einem Kind unter drei Jahren vor, wenn es in einer Raumordungsregion lebt, in der ein Platz in öffentlich geförderter Kindertagesbetreuung für weniger als ein Drittel der Kinder gesichert ist. Eine Rationierung liegt jedoch nicht vor, wenn das Kind bereits im Vorjahr einen Platz in öffentlich geförderter Kindertagesbetreuung hatte. Nach dieser Definition betrifft eine Rationierung etwa 87 Prozent der unter 3-Jährigen.

Zu den Variablen, die gemäß unseren Schätzergebnissen die Nachfrage nach öffentlicher Betreuung signifikant erhöhen, zählen ein hoher Bildungsabschluss der Mutter (Abitur), das Alter des Kindes, Geschwister im Kindergarten, sowie ein Wohnort in den ostdeutschen Bundesländern. Weitere Kinder im Haushalt senken die Wahrscheinlichkeit, dass Eltern externe Kinderbetreuung in Anspruch nehmen möchten. In der Angebotsgleichung zeigen sich nur wenige systematische Einflüsse. Allerdings kommt das niedrigere Betreuungsangebot für jüngere Kinder verglichen mit dem Angebot für ältere Kinder klar heraus.

Die Schätzung der erwarteten Kinderbetreuungskosten lässt sich anhand einiger Kennziffern zusammenfassen. Prognostiziert man die Kosten anhand der beobachteten individuellen Merkmale, ergibt sich im Mittel über alle Haushalte ein Monatsbetrag pro Kind von 143 Euro bei Ganztagesbetreuung und 80 Euro bei Halbtagesbetreuung. Die Wahrscheinlichkeit, dass ein Haushalt, der öffentliche externe Kinderbetreuung nachfragt, diese nicht erhält, wird für jeden Haushalt unter Berücksichtigung der Fehlerterme des „Partial Observability Modells“ simuliert und beträgt im Mittel über die Familien mit Kindern unter 3 Jahren bei 25\%.

Bei der Schätzung des Verhaltensmodells zur simultanen Wahl von Arbeitsangebot und Kinderbetreuung fokussieren wir auf Paarhaushalte, bei denen Haushaltsvorstand und Partner zwischen 20 und 65 Jahren alt sind, und für die die relevanten sozio-ökonomischen Merkmale beobachtet werden. ${ }^{9}$ Es gehen 12.073 Verhaltensbeobachtungen ein, davon 3.158 Beobachtungen von Eltern mit Kindern. Durch die Einbeziehung der Beobachtungen aus Haushalten, die lediglich über ihr Arbeitsangebot entscheiden, werden die Parameter des Angebotsmodells präziser geschätzt. In der Stichprobe fallen bei etwa der Hälfte der Haushalte mit Kindern unter 6 Jahren keine Kinderbetreuungskosten an, da keine (oder kostenfreie) externe Betreuung genutzt wird. Von den extern betreuten Kindern ist rund ein Viertel in Ganztagesbetreuung. Etwa 60\% der Mütter sind nicht erwerbstätig und nur 15\% Vollzeit beschäftigt.

\footnotetext{
${ }^{9}$ Die Fallzahlen bei den Alleinerziehenden reichten nicht aus, um für diesen Familientyp ein um ein Kinderbetreuungsmodul erweitertes Modell zu schätzen. Ein traditionelles diskretes Arbeitsangebotmodell ist wenig aussagekräftig, weil das Betreuungsgeld einen reinen Einkommenseffekt erzeugt, von dem erfahrungsgemäß
} 
Die geschätzten Parameter des Verhaltensmodells implizieren, dass Eltern eine starke Präferenz haben, ihre Kinder selbst zu betreuen. Der Indikator für eine intensive eigene Betreuung geht signifikant positiv, die Indikatoren für externe Halbtages- oder Ganztagesbetreuung signifikant negativ, in die empirische Nutzenfunktion ein. Nachdem die Kinderbetreuungszeit als endogene Entscheidungsvariable aufgefasst wird, zeigt sich für Mütter mit Kleinkindern keine signifikant höhere Freizeitpräferenz. Die Freizeitpräferenz und damit die Neigung, nicht am Arbeitsmarkt teilzunehmen, ist für westdeutsche Frauen allerdings signifikant höher als für ostdeutsche Frauen. Hervorzuheben ist auch eine negative Interaktion der Freizeit des Mannes mit der Freizeit der Frau. Das deutet daraufhin, dass die Aktivitäten der Ehepartner eher substituierbar sind, d.h. der Nutzen des Haushalts wächst bei Spezialisierung eines Partners auf Marktarbeit. Hinsichtlich des Nutzens aus dem verfügbaren Einkommen zeigt sich, dass Haushalte mit Kleinkindern den Konsum systematisch niedriger gewichten.

\subsection{Annahmen zur Simulation}

Das mikroökonometrisch geschätzte Verhaltensmodell lässt sich nutzen, um Verhaltensänderungen bei Veränderungen exogener Modellgrößen abzuschätzen. Dazu muss die Annahme getroffen werden, dass die Veränderung dieser Größen die geschätzten strukturellen Präferenzparameter des Haushaltsverhaltens nicht beeinflusst. Auch die haushaltspezifischen Charakteristika, zum Beispiel die Kinderzahl, müssen als gegeben angenommen werden. Anders ausgedrückt, darf eine zu analysierende Reform lediglich die Beschränkungen oder institutionellen Rahmenbedingungen, unter denen die Haushalte ihre Entscheidungen treffen, berühren.

Die Verhaltensreaktion wird demnach durch einen Impuls auf das Einkommen ausgelöst, das bei Entscheidung für eine der zugelassenen Handlungsmöglichkeiten jeweils zur Verfügung steht. Als Reaktion auf diesen Impuls lassen sich für jeden Haushalt individuelle Wahrscheinlichkeiten angeben, dass der Haushalt sein Verhalten gegenüber dem Basisszenario ändert. Eine Verhaltensänderung kommt zustande, wenn durch die Einführung des Betreuungsgeldes, eine andere Wahloption als die im Basisszenario gewählte Option den höchsten Nutzen stiftet. Weil dieser Effekt auf der Haushaltsebene berechnet wird, muss für Aussagen auf der Ebene der Bevölkerung eine Hochrechnung vorgenommen werden. Diese beruht auf Hochrechnungsfaktoren, die beschreiben, bei wie vielen Haushalten in der Gesamtbevölkerung eine

kaum Verhaltensanpassungen ausgehen. Dies bestätigten Simulationsrechnungen für Alleinerziehende in einem entsprechend reduzierten Modell. 
Kombination von sozio-ökonomischen Merkmalen vorkommt, die einem Haushalt aus der Stichprobe entspricht.

Für die Simulation der Wirkungen des Betreuungsgeldes sind zwei Einkommensimpulse zu berücksichtigen. Erstens muss die geplante Ausweitung des Angebots an öffentlich geförderter Kindertagesbetreuung abgebildet werden, da der Vorschlag eines Betreuungsgeldes eine Folge der sich verändernden Wahlmöglichkeiten ist. Bei einem Ausbau der öffentlich geförderten Kindertagesbetreuung reduziert sich die Wahrscheinlichkeit, dass Eltern, die diese Betreuung nachfragen, rationiert sind. Hierdurch sinken die erwarteten Kosten der Kinderbetreuung, da ein Ausweichen auf eine private Organisation bei Rationierung annahmegemäß teurer ist. Dies wiederum beeinflusst über die Veränderung des verfügbaren Haushaltseinkommens Art und Umfang der Kinderbetreuung und das Arbeitsangebot. Unsere Simulationen basieren auf der Annahme, dass zur Einführung des Betreuungsgeldes genügend Plätze in öffentlich geförderter Kindertagesbetreuung verfügbar sind. Die bestehende Rationierung der Elternnachfrage nach externer Kinderbetreuung wird also vollständig aufgehoben.

Zweitens verändert das Betreuungsgeld das verfügbare Einkommen unabhängig vom gewählten Arbeitsangebot, jedoch abhängig von der gewählten Form der Kinderbetreuung. Konkret unterstellen wir in Anlehnung an die in Thüringen geltenden Regeln, dass Eltern Anspruch auf ein Betreuungsgeld von monatlich 150 Euro haben, wenn sich mindestens ein zu betreuendes Kind im Alter von 13 bis 36 Monaten im Haushalt befindet und das Kind keine öffentlich geförderte Kindertagesbetreuung besucht. Anders als in Thüringen (vgl. Abschnitt 2.2) wird das Betreuungsgeld in unserem Modell für jedes Kind, das die Bedingungen erfüllt, in gleicher Höhe ausbezahlt. Befinden sich mehrere Kinder im Alter zwischen einem und drei Jahren im Haushalt, erhält der Haushalt das Betreuungsgeld für jedes Kind. Darüber hinaus wird angenommen, dass das Betreuungsgeld kein zu versteuerndes oder anzurechnendes Einkommen darstellt, sondern pauschal ausbezahlt wird. Dieses Design führt dazu, dass die eigene Betreuung im Verhältnis zu den anderen im Modell zugelassenen Formen externer Kinderbetreuung prinzipiell attraktiver wird. Wie stark die ausgelösten (simulierten) Verhaltensänderungen sind, ist jedoch eine empirische Frage, die wir im folgenden Abschnitt klären. 


\section{Simulationsergebnisse}

\subsection{Arbeitsangebot}

Die zu simulierenden Reformwirkungen auf das Arbeitsangebot setzen sich aus zwei Effekten zusammen: den Folgen der Einführung des Betreuungsgeldes und der Angebotsausweitung im Bereich öffentlicher Kindertagesbetreuung. Während die Wirkungen auf das Arbeitsangebot der Väter (hier wie auch später) zu vernachlässigen sind, zeigen sich bei den Frauen spürbare Wirkungen. ${ }^{10}$ So senkt die Einführung des Betreuungsgeldes bei rationiertem Angebot an öffentlich geförderter Kinderbetreuung die Partizipationsrate um 8,3 Prozentpunkte (Tabelle $1)$.

\section{(Tabelle 1 ungefähr hier)}

Annähernd die Hälfte der Mütter in Teilzeit hört auf zu arbeiten. Diese Gruppe erzielt vielfach nur ein geringes Erwerbseinkommen, so dass das Betreuungsgeld und der Wegfall der Betreuungskosten den Abfall beim verfügbaren Einkommen relativ gut kompensieren. Zudem entsteht ein Nutzengewinn aus mehr eigener Betreuung. Betrachtet man die Matrix der Übergänge zwischen den verschiedenen Zuständen, zeigt sich, dass die Einführung des Betreuungsgelds Mütter in Vollzeit im Gegensatz dazu kaum betrifft. ${ }^{11}$ In diesen Familien - überwiegend Doppelverdiener-Haushalten - wäre der Wert des Betreuungsgeldes im Vergleich zum Familieneinkommen relativ gering. Der Einkommenseffekt ist zu klein, um eine Verringerung des Arbeitsangebots auszulösen. Hier wirkt sich aus, dass das Betreuungsgeld pauschaliert - und damit unabhängig vom Arbeitsangebot bzw. Erwerbsstatus in der Ausgangslage - gestaltet ist. Aus diesem Grund wirkt die Leistung auf Frauen mit höheren Bildungsabschlüssen deutlich schwächer.

Betrachtet man eine isolierte Ausweitung der Kinderbetreuung ohne Betreuungsgeld, steigt das Arbeitsangebot der Frauen. Die Rate der Nichtpartizipation fällt bei nachfragegerechter Verfügbarkeit von Betreuungsmöglichkeiten um 3,6 Prozentpunkte. Die Rate der Frauen in Vollzeit steigt stärker (um 4,1 Prozentpunkte), weil auch Teilzeit arbeitende Frauen in Voll-

\footnotetext{
${ }^{10}$ Für die hier berichteten Ergebnisse ist grundsätzlich zu berücksichtigen, dass bei einer ex ante Analyse Ungenauigkeiten aufgrund der Schätzung sowie der anschließenden Prognose auftreten. In Mikrosimulationsmodellen lassen sich Konfidenzintervalle, mit denen die Signifikanz der Effekte im statistischen Sinne beurteilt werden kann, prinzipiell mit Bootstrap- Methoden berechnen. Weil dies im Rahmen des hier genutzten komplexen Modells mit ganz erheblichem Aufwand verbunden wäre, müssen wir auf entsprechende Angaben verzichten. Diese Vorgehensweise ist in der Literatur zur ex ante Evaluation allerdings nicht untypisch (vgl. Blundell et al., 2009 und Creedy et al., 2007).
} 
zeit wechseln. Durch eine Reduktion der erwarteten Kosten bei externer Betreuung lassen sich klar positive Arbeitsangebotswirkungen erzielen. Die Maßnahme begünstigt Frauen mit hoher Erwerbsneigung, etwa Hochqualifizierte, systematisch stärker.

Werden Betreuungsgeld und Ausbau der Kinderbetreuung simultan betrachtet, verändern rund 13\% der Mütter mit Kleinkindern ihr Arbeitsangebot: 5,1\% erhöhen es, 8,1\% verringern es. Die erwerbstätigen Frauen, die ein Betreuungsgeld in Anspruch nehmen und aufhören zu arbeiten, kehren auch nicht auf den Arbeitmarkt zurück, falls die öffentlich geförderte Kinderbetreuung ausgebaut wird. Die Mütter mit hoher Erwerbsneigung, die vom Abbau der Rationierung profitieren, ziehen sich auch nicht vom Arbeitsmarkt zurück, wenn das Betreuungsgeld eingeführt wird. So wächst die Polarisierung am Arbeitsmarkt, zu Lasten der Mütter in Teilzeit. Ihr Anteil an den Müttern mit Kindern im Alter von 13 bis 36 Monaten fällt von 14,7 auf 6,8\%. Hierbei spielt die Kinderzahl eine wichtige Rolle. Frauen mit zumindest einem weiteren Kind unter sechs Jahren ziehen sich nach der Reform besonders häufig vom Arbeitsmarkt zurück. $^{12}$

\section{(Tabelle 2 ungefähr hier)}

Tabelle 2 beleuchtet die unterschiedlichen Wirkungen der Kombination von Betreuungsgeld und Ausbau der Kinderbetreuung für verschiedene Gruppen von Müttern genauer. Bei Alleinverdienerhaushalten fördert die Maßnahme ausschließlich die Erwerbsbeteiligung, während das Bild in Zweiverdienerhaushalten gespalten ist. Mütter, die als Zweitverdiener ein relativ hohes Einkommenspotenzial haben, wechseln von Teilzeit zu Vollzeit. Ist der Zweitverdienst, wie in der Mehrheit der Fälle, nur eine geringfügige Beschäftigung, dominiert der Übergang zur Nicht-Partizipation. Da geringfügige Beschäftigung bei geringer Qualifizierten stärker ausgeprägt ist, ist der Rückzug vom Arbeitsmarkt (die Ausweitung des Arbeitsangebots) relativ häufiger (seltener) als bei den besser Qualifizierten. Dennoch zeigt sich hinsichtlich der Einkommensposition des Haushalts im Status quo ein bemerkenswertes Muster. Bei Haushalten im untersten Einkommensquartil dominiert der positive Arbeitsangebotseffekt des Aus-

\footnotetext{
${ }^{11}$ Die Matrix der Übergänge kann auf Nachfrage von den Autoren zur Verfügung gestellt werden.

${ }^{12}$ Sollte der Ausbau der öffentlich geförderten Kinderbetreuung die Rationierung nicht vollkommen aufheben, so ist zu erwarten, dass Mütter mit hoher Erwerbsneigung ihr Arbeitsangebot in geringerem Maße ausweiten können. Die negativen Effekte des Betreuungsgeldes würden hingegen weiterhin bestehen. Insgesamt müsste dann mit negativeren Arbeitsangebotseffekten gerechnet werden.
} 
baus der Kinderbetreuung. ${ }^{13}$ Diese können wegen Liquiditätsbeschränkungen eine Rationierung nicht durch Ausweichen auf ungeförderte private externe Betreuungslösungen überwinden. Darum wirkt die Senkung der Betreuungskosten durch den Ausbau der öffentlich geförderten Angebote in dieser Personengruppe besonders stark. Bei Haushalten im mittleren Einkommenssegment (2. und 3. Quartil) dominiert dagegen der negative Effekt des Betreuungsgeldes. Sie können sich einen gewissen Erwerbseinkommensverlust durch Rückzug vom Arbeitsmarkt leisten, wenn dieser durch ein Betreuungsgeld teilweise kompensiert wird.

\subsection{Nachfrage nach öffentlich geförderter Kindertagesbetreuung}

Die simulierten Effekte auf die Nachfrage nach öffentlich geförderter Kindertagesbetreuung sind stark mit den Arbeitsangebotseffekten korreliert. Erneut erlegen wir die Wirkungen der Reform in zwei Komponenten. Wird das Betreuungsgeld isoliert eingeführt, fällt die Nachfrage nach externer Kinderbetreuung drastisch. Der Anteil der Haushalte, die ihre Kinder nicht in Kindertageseinrichtungen betreuen lassen, wächst um 8,2 Prozentpunkte auf annähernd 70 Prozent (Tabelle 3). Der Rückgang ist bei den Kindern in Halbtagesbetreuung besonders stark: mehr als jedes vierte Kind wird nicht länger extern betreut, wenn die Betreuung daheim finanziell gefördert wird. Dahinter steht der oben beschriebene Rückzug der in Teilzeit bzw. nur geringfügig beschäftigten Mütter vom Arbeitsmarkt. Bei den Kindern in Ganztagesbetreuung, deren Mütter vielfach stärker am Arbeitsmarkt engagiert sind, betrifft eine solche Verhaltensänderung weniger als jedes zehnte Kind.

\section{(Tabelle 3 ungefähr hier)}

Simuliert man die Wirkungen eines Ausbaus der Kinderbetreuung isoliert, zeigen sich klar gegenläufige Wirkungen. Die Überwindung der Rationierung bei einem nachfrageorientierten Angebot steigert die Rate der Inanspruchnahme externer Betreuungslösungen von 38,7 auf 43,2\%. Davon profitiert mit einem Zuwachs um fast 30 Prozent vor allem die Ganztagesbetreuung. Dieser Zuwachs speist sich überwiegend aus Haushalten, die im Status quo daheim betreuen. Es bestätigt sich, dass die derzeitige Rationierung insbesondere bei Betreuungsmöglichkeiten besteht, die Müttern auch eine Vollzeittätigkeit gestatten würden.

\footnotetext{
${ }^{13}$ Die Einkommenskategorisierung wird auf Basis der Haushaltsäquivalenzeinkommen vorgenommen; das Nettoeinkommen wird entsprechend der Personen im Haushalt gewichtet. Im ersten Einkommensquartil kann es keinen Übergang von Teilzeit zu Vollzeit geben, da hier keine Mutter im Status Quo in Teilzeit arbeitet.
} 
Vergleicht man die Wirkungen der beiden Einzelmaßnahmen, erscheinen die Effekte des Ausbaus der öffentlichen Betreuungsangebote, also einer Steigerung des verfügbaren Einkommens durch sinkende externe Betreuungskosten, etwas schwächer als die Wirkungen der Einkommensverbesserung durch ein Betreuungsgeld. Entsprechend dominiert in der Gesamtbetrachtung der Effekt des Betreuungsgeldes. Der Anteil der Kinder, die daheim betreut werden, steigt um 4,1 Prozentpunkte. Insgesamt verliert die Halbtagesbetreuung deutlich an Gewicht, während die Ganztagesbetreuung in öffentlich geförderten Einrichtungen trotz der belastenden Einflüsse des Betreuungsgeldes noch um ein Fünftel zunimmt. ${ }^{14}$

\section{(Tabelle 4 ungefähr hier)}

Das Betreuungsgeld bei bedarfsgerechtem Ausbau der Kindertagesbetreuung bewirkt bei 8,1\% der Haushalte mit Kindern in der relevanten Altersgruppe eine Abnahme der externen Betreuung, bei 5\% eine Zunahme. Betrachtet man die Wirkungen für verschiedene Gruppen von Müttern, zeigt sich eine ähnliche Heterogenität wie bei den Arbeitsangebotseffekten (vgl. Tabelle 2). So reduzieren immerhin 20,8\% der Zweiverdiener-Haushalte die externe Kinderbetreuung, nur 5,7\% weiten sie aus. Bei den Alleinverdiener-Haushalten, bei denen externe Kinderbetreuung im Status quo eine untergeordnete Rolle spielt, sind dagegen kaum Effekte zu erwarten. Bei Müttern mit niedriger Bildung halten sich positive und negative Wirkungen in etwa die Waage: 9,5\% reduzieren, 6,7\% erhöhen den Umfang der externen Betreuung. Hierbei kommt wiederum der relativ starke positive Effekt bei den Müttern im untersten Einkommensquartil zum Tragen, bei denen eine Rationierung die Realisation des gewünschten Arbeitsangebots spürbar dämpft.

Ähnlich sind auch die positiven Wirkungen bei ausländischen Müttern zu erklären, die häufig gering qualifiziert und einkommensschwach sind. In dieser Bevölkerungsgruppe weiten 5,1\% die externe Betreuung aus, während nur 2,9\% weniger externe Betreuung nutzen. Offenbar ist hier die bestehende Rationierung als Erklärungsfaktor einer niedrigen Erwerbsbeteiligung besonders bedeutsam. Bei den Familien im obersten Einkommenssegment sind die monetären Anreize des Betreuungsgeldes praktisch unwirksam. Hier nehmen zusätzlich 6,8\% der Haushalte bei einem verbesserten Angebot eine externe Betreuung auch wahr, aber nur 1,7\% nutzen das Betreuungsgeld, um selber für Betreuung zu sorgen.

\footnotetext{
${ }^{14}$ Sollte der Ausbau der öffentlich geförderten Kinderbetreuung die Rationierung nicht vollkommen aufheben, würde dies vermutlich vor allem zu Lasten der Ganztagesbetreuung gehen. Die Zunahme der Ganztagesbetreuung würde dann geringer ausfallen, während die Halbtagesbetreuung aufgrund des Betreuungsgeldes in ähnlichem Maße zurückgehen würde.
} 


\subsection{Zeit für Kinder}

Während ein Betreuungsgeld in Kombination mit einem nachfragegerechten Angebot öffentlich geförderter Kinderbetreuung spürbare negative Wirkungen auf das Arbeitsangebot und die Häufigkeit externer Betreuung entfaltet, zeigt die Simulation nur marginale Wirkungen auf die Betreuungszeiten innerhalb der Familie. Bei weniger als einem Prozent der Frauen mit Kindern in der relevanten Altersgruppe beobachten wir eine Veränderung der selbst geleisteten Betreuungsstunden. Demnach lassen auch Mütter, die das Betreuungsgeld in Anspruch nehmen bzw. vom Ausbau der externen Betreuungsmöglichkeiten profitieren, die ihren Kindern gewidmete Betreuungszeit unverändert.

Dieses Ergebnis erscheint überraschend. Eine Erklärung ist jedoch, dass die Reformen überwiegend das Entscheidungsverhalten von Müttern ändern, die im Status quo Teilzeit und hier vielfach nur geringfügig beschäftigt sind. Dieses Erwerbsmuster erlaubt schon in der Ausgangslage intensive eigene Kinderbetreuung, so dass sich der innerfamiliäre Betreuungsstatus im Modell nicht ändert. Eine weitere Erklärung ist, dass die Mütter eigene Kinderbetreuung in der Nutzenfunktion im Vergleich zum sonstigen Konsum relativ hoch gewichten. Dies macht den theoretisch zu erwartenden Substitutionseffekt schwach: die Nutzengewinne aus fallenden Kinderbetreuungskosten sind im Verhältnis zum Nutzenverlust aus weniger eigener Kinderbetreuung klein. Anders ausgedrückt müsste der Preis externer Kinderbetreuung noch sehr viel stärker sinken, um eigene Betreuungsarbeit klarer zu verdrängen. Im Übrigen zeigt die Simulation die zu erwartende Neuordnung des Kinderbetreuungsarrangements bei Familien, die gegenwärtig durch ein zu kleines öffentliches Angebot rationiert sind. Immerhin jede dritte Mutter, die nach Ausbau der öffentlich geförderten Kinderbetreuung eine Erwerbstätigkeit beginnt, reduziert dafür den eigenen Betreuungsumfang. Diese Frauen stellen jedoch 0,8\% der Mütter mit Kindern in der relevanten Altersgruppe, so dass der Effekt über alle Haushalte betrachtet praktisch nicht relevant ist. ${ }^{15}$

Allerdings muss einschränkend auch betont werden, dass das Raster der in unserem Mikrosimulationsmodell zugelassenen eigenen Kinderbetreuungszeiten - „hoch“ und „niedrig“ - wegen Datenbeschränkungen relativ grob ist. ${ }^{16}$ Entsprechend sind relativ starke externe Impulse nötig, um überhaupt einen Übergang zwischen den Alternativen auszulösen.

\footnotetext{
${ }^{15}$ Der niedrige Anteil führt zu niedrigen Fallzahlen, so dass die statistische Unsicherheit an dieser Stelle hoch ist.

${ }^{16}$ Eine hohe mütterliche Kinderbetreuungszeit umfasst in unserem Modell 35 Stunden pro Woche. Die niedrige Kinderbetreuungszeit entspricht 16 Stunden pro Woche. Siehe Abschnitt 3.1.
} 


\subsection{Verfügbares Haushaltseinkommen}

Die analysierte Reform kann das verfügbare Haushaltseinkommen direkt und indirekt beeinflussen. Erstens hat die Auszahlung des Betreuungsgeldes - bei Inanspruchnahme - einen direkten Effekt. Zweitens entsteht für Familien, die im Status quo durch ein zu geringes Angebot an öffentlich geförderter Kindertagesbetreuung rationiert sind, ein Einkommensgewinn, weil der Preis für externe Betreuung fällt. Die Ersparnis durch öffentliche statt privater Betreuung beträgt pro Woche 167 Euro (82 Euro) je Kind in Ganztagesbetreuung (Halbtagesbetreuung). Drittens ändert sich das Einkommen der Familien, deren Verhalten auf die Reform reagiert: Veränderungen im Arbeitsangebot berühren das Erwerbseinkommen, Veränderungen beim Betreuungsarrangement die Kosten der Kinderbetreuung. ${ }^{17}$

(Tabelle 5 ungefähr hier)

Tabelle 5 fasst die simulierten Einkommenswirkungen zusammen. Im Durchschnitt über alle Haushalte mit Kindern in der relevanten Altersgruppe steigert die Reform das verfügbare Einkommen um 118 Euro. Der weitaus überwiegende Teil ist dabei direkten Einkommenseffekten zuzurechnen, die ohne Verhaltensänderung zustande kommen und daher als Mitnahmeeffekte interpretiert werden können. Allein durch das Betreuungsgeld wächst das verfügbare Haushaltseinkommen in der Zielgruppe um durchschnittlich 115 Euro monatlich. Über alle Haushalte hinweg heben sich die Konsumgewinne durch den Abbau der Rationierung und die Konsumverluste durch die Reduktion der Arbeitszeiten annähernd auf.

Allerdings hängen die Einkommenswirkungen der Reform stark vom Erwerbsstatus der Mutter in der Ausgangslage ab. Bei den nicht Erwerbstätigen beanspruchen fast alle Familien ohne Verhaltensänderung - das Betreuungsgeld, denn die Nutzung externer Betreuungsmöglichkeiten ohne Erwerbstätigkeit der Mutter ist bei Familien mit Kindern unter 3 Jahren sehr selten. Die zusätzliche Einkommensverbesserung durch längere Arbeitszeiten (18 Euro) und der Einkommensverlust durch Inanspruchnahme von mehr externer Betreuung (2 Euro) nehmen sich dagegen klein aus. Allerdings wechseln nur 5\% der nicht erwerbstätigen Mütter in die Erwerbstätigkeit. Für ihre Familien steigt das Arbeitseinkommen um durchschnittlich 389 Euro. Nach Abzug der Kosten der Kinderbetreuung bleibt ein Gewinn an verfügbarem Einkommen von 167 Euro.

\footnotetext{
${ }^{17}$ Ein weiterer Effekt folgt aus möglichen fiskalischen Rückwirkungen. Die Maßnahmen müssen finanziert werden, so dass sich indirekt die Steuerlast ändern kann. Mit größeren gesamtwirtschaftlichen Rückwirkungen über
} 
Ein anderes Muster zeigt sich bei den im Status quo erwerbstätigen Müttern. In dieser Gruppe lässt die Reform das Einkommen insgesamt praktisch unverändert. Verantwortlich für dieses Ergebnis ist der starke Rückzug aus Teilzeitbeschäftigungen, verbunden mit substanziellen Erwerbseinkommenseinbußen (65 Euro). Diese werden durch das Betreuungsgeld, sowie die Gewinne aus weniger und günstigerer externer Kinderbetreuung, im Durchschnitt gerade kompensiert.

Die Reform verteilt demnach, von der Seite des verfügbaren Einkommens her betrachtet, zu Gunsten von Haushalten um, die heute nicht arbeiten und darum die neue familienpolitische Leistung, ohne ihre Erwerbs- und Betreuungsentscheidung ändern zu müssen, mitnehmen können. Diese Umverteilung steht mit dem Ziel einer finanziellen Besserstellung potenziell benachteiligter Gruppen durchaus im Einklang. Eine Analyse der Einkommenswirkungen der Reform nach Haushaltsmerkmalen zeigt (vgl. Tabelle 5, unterer Teil), dass im Durchschnitt Ausländer stärker als Deutsche, Mütter mit niedriger Bildung stärker als Mütter mit hoher Bildung, Haushalte im untersten Einkommensquartil stärker als Haushalte im obersten Einkommensquartil profitieren. Dieses Ergebnis setzt allerdings voraus, dass das Betreuungsgeld nicht auf die Leistungen der sozialen Grundsicherung, insbesondere das Arbeitslosengeld II, angerechnet wird, wie es aus systematischen Gründen eigentlich geboten wäre.

\subsection{Fiskalische Kosten}

Die fiskalischen Kosten der Reform lassen sich nicht unmittelbar aus dem Modell hochrechnen. Zum einen sind die Kosten des Ausbaus der Kinderbetreuung selbst nicht abgebildet. Zum anderen ist das Betreuungsgeld nicht heute, sondern erst nach dem für 2013 anvisierten Ausbau der Kinderbetreuung zu implementieren. Bis dahin verändert sich gegenüber dem Status quo durch demografische Veränderungen die Zahl der Geburten jedoch merklich. Gemäß der 11. koordinierten Prognose des Statistischen Bundesamtes, ist für das Jahr 2013 mit nur noch rund 600.000 Geburten zu rechnen. In Verbindung mit der im Modell simulierten Quote der Inanspruchnahme des Betreuungsgelds von rund zwei Drittel folgen daraus direkte fiskalische Kosten von 1,4 Mrd. Euro jährlich.

Die Einsparungen durch eine geringere Nachfrage von öffentlich geförderten Kinderbetreuungsplätzen als Folge des Betreuungsgeldes sind hier nicht mit eingerechnet. Würde das

Löhne und Arbeitslosigkeit ist dagegen eher nicht zu rechnen. Die Analyse makroökonomischer Rückkopplungen sprengt aber den gewählten analytischen Rahmen. 
Betreuungsgeld nicht eingeführt, bestünde nämlich auch bei Ausbau des Versorgungsgrades bei den 1- bis 3-Jährigen auf ein Drittel die Rationierung bei der Kinderbetreuung weiter. Mit dem Betreuungsgeld fällt der Bedarf dagegen auf etwa 35\%, so dass sich Angebot und Nachfrage so gut wie ausgleichen.

Zusätzlich zu den direkten Kosten des Betreuungsgeldes müssen Einnahmeausfälle durch das fallende Arbeitsangebot in Rechnung gestellt werden. Die Nettokosten durch entgangene Einkommensteuer (113 Mio. Euro jährlich) und Sozialversicherungsbeiträge (212 Mio. Euro jährlich) sind jedoch, wie die Arbeitsangebotseffekte selbst, moderat, zumal viele Mütter ihr Arbeitsangebot aus einer steuer- und beitragsbegünstigten geringfügigen Beschäftigung heraus anpassen.

\section{$5 \quad$ Zusammenfassende Bewertung}

Geht man von den internationalen Erfahrungen aus, ist zu erwarten, dass ein Betreuungsgeld auch in Deutschland zu spürbaren Verhaltensänderungen führen könnte. In Finnland und Norwegen hat ein Betreuungsgeld die Inanspruchnahme öffentlich geförderter Betreuungseinrichtungen tendenziell verringert. Damit einhergehend ist die Erwerbsbeteiligung der betreuenden Mütter leicht gefallen. Allerdings lassen sich diese Erfahrungen schon wegen der anderen familienpolitischen Systeme in Skandinavien nicht ohne Weiteres auf Deutschland übertragen. Da Ursache-Wirkungs-Zusammenhänge für das Betreuungsgeld in Thüringen bei derzeitiger Datenlage nicht ex post geschätzt werden können, nutzt diese Studie das Instrument der Mikrosimulation, um die potenziellen Wirkungen eines Betreuungsgeldes bei gleichzeitigem Ausbau der Kinderbetreuung in Deutschland ex ante abzuschätzen.

Gemäß unserer Simulation würden bei einem bedarfsgerechten Ausbau der Kindertagesbetreuung die Inanspruchnahme und das Arbeitsangebot moderat wachsen. Die mit dem Ausbau anvisierte Betreuungsquote von einem Drittel entspricht dabei in etwa dem Bedarf, da bei den Müttern mit Kindern unter 3 Jahren die Neigung zu einer eigenen Betreuung stark ist. Dennoch beginnen nach Ausbau der Betreuungsmöglichkeiten mindestens fünf Prozent der Mütter eine berufliche Tätigkeit, überwiegend sogar in Vollzeit. Das Betreuungsgeld selbst führt zu einer moderaten Abnahme des Arbeitsangebots der Mütter und der Nachfrage nach externer Kinderbetreuung.

Die Gruppe von Familien, die so auf das Betreuungsgeld reagieren, ist dabei eine andere als die Gruppe von Familien, die auf den Ausbau der Kinderbetreuung reagiert. Insbesondere 
teilzeitbeschäftigte Mütter sind bereit, ihre berufliche Tätigkeit aufzugeben und sich selbst um ihre Kinder zu kümmern. Geschätzt beinahe jede zweite, der vor Einführung des Betreuungsgeldes, Teilzeit arbeitende Mutter würde sich vom Arbeitsmarkt zurückziehen. Allerdings stellt diese Gruppe kaum sieben Prozent der Mütter mit Kindern in der relevanten Altersgruppe. Vollzeitbeschäftigte Mütter, oft mit besserem Bildungshintergrund und in guter Einkommensposition, werden vom Betreuungsgeld dagegen kaum berührt. Die Einkommenseinbußen bei Rückzug vom Arbeitsmarkt wären deutlich höher als das erhaltene Betreuungsgeld, sodass die Inanspruchnahme der Leistung unattraktiv ist.

Die insgesamt moderaten Verhaltenseffekte des Betreuungsgeldes erklären sich hauptsächlich aus drei Gründen: Erstens ist das Betreuungsgeld als Pauschalleistung gestaltet und nicht von Einkommen, Erwerbsstatus oder dem Umfang der ausgeübten Tätigkeit abhängig. So ausgestaltete Transfers entfalten allgemein nur marginale Auswirkungen auf das Arbeitsangebot. Zweitens ist die Höhe der Transferleistung für viele Haushalte im Verhältnis zum Haushaltseinkommen relativ gering. Ein schwacher finanzieller Impuls wird kaum substanzielle Verhaltenseffekte verursachen. Drittens führt die niedrige Erwerbsneigung von Müttern mit Kindern in der relevanten Altersgruppe selbst nach Ausbau der öffentlich geförderten Kindertagesbetreuung zu einer hohen Quote familiärer Betreuung. Bei Familien, die sich bereits für eine Betreuung durch die Mutter entschieden haben, kann das Betreuungsgeld keine Verhaltenseffekte mehr auslösen.

Anders interpretiert, sind die pro Jahr für das Betreuungsgeld insgesamt aufzuwendenden ein bis zwei Mrd. Euro jährlich eher als Mittel der Umverteilung finanzieller Ressourcen hin zu einkommensschwächeren Haushalten anzusehen. Das Betreuungsgeld fördert somit das Alleinverdienermodell. Dabei sollten die Verteilungswirkungen des Betreuungsgeldes nicht nur am laufenden Haushaltseinkommen festgemacht werden. Hinzu treten ungünstige dynamische Effekte, die sich im hier verwendeten kurzfristigen Simulationsmodell nicht abbilden lassen. $\mathrm{Zu}$ bedenken ist zum einen die Einkommensdynamik nach geburtenbedingten Erwerbsunterbrechungen. Zum anderen müssen bei der Beurteilung eines Betreuungsgeldes angesichts der wenig ausgeprägten Bildungsmobilität in Deutschland die längerfristigen Verteilungswirkungen, die von der Qualität der Kinderbetreuung im Kleinkindalter ausgehen, bedacht werden (Pfeiffer/Reuß 2008).

Die ungünstigen Folgen längerer Erwerbsunterbrechungen aufgrund von Erziehungszeiten für Beschäftigungschancen und Löhne von Frauen sind empirisch gut dokumentiert. Beblo/Wolf 
(2002) zeigen für Deutschland signifikante Lohneinbußen beim Wiedereinstieg von Müttern in den Arbeitsmarkt, wenn der Erwerbsverlauf vollständig unterbrochen wird. Dagegen ist bei Rückkehrerinnen aus Teilzeit kein systematischer Lohnabschlag zu beobachten. Insofern könnte der durch das Betreuungsgeld geförderte Rückzug vom Arbeitsmarkt bei bisher in Teilzeit tätigen Müttern kontraproduktiv wirken. Hinzu kommen mittel- und langfristige negative Effekte auf spätere Karrierechancen, falls die Verringerung des Arbeitsangebots die Dauer der Elternzeit verlängert, also den Wiedereinstieg in den Arbeitsmarkt verzögert (Ziefle, 2004). Zu erwarten ist, dass die Mütter, die auf das Betreuungsgeld reagieren, es auch über die maximal mögliche Zeit von zwei Jahren ausschöpfen.

Noch bedeutsamer sind möglicherweise die ungünstigen Folgen des Betreuungsgeldes für die frühkindliche Bildung. Zwar sind die Wirkungen von frühkindlicher Betreuung in Kindertageseinrichtungen auf den späteren Schul- und Arbeitsmarkterfolg in Deutschland bisher kaum systematisch untersucht. Die internationale Evidenz legt jedoch eindeutig nahe, dass die Inanspruchnahme qualitativ hochwertiger Kindertagesbetreuung außerhalb des Elternhauses bei Kindern aus sozioökonomisch benachteiligten Familien positiv auf die kognitive und sozioemotionale Entwicklung wirkt. Dies wiederum begünstigt nachhaltig den späteren Bildungsund Arbeitsmarkterfolg (Biedinger/Becker 2006).

Insofern erscheint es bedenklich, dass das Betreuungsgeld gerade Familien mit niedrigem Bildungsstand oder mit Migrationshintergrund besonders gut erreicht. Es stärkt in diesen Gruppen noch die bereits bestehende Neigung, Kleinkinder innerhalb der Familie zu betreuen. Anders gewendet schafft das Betreuungsgeld neue Hürden, in eine externe Kindertagesbetreuung zu kommen, gerade für Kinder, die davon besonders profitieren könnten. Darum sollte genau der umgekehrte Ansatz bedacht werden: Eine Politik, die bei bildungsfernen Familien eine qualitativ hochwertige frühkindliche Betreuung in öffentlich geförderten Einrichtungen unterstützt, könnte langfristig mehr zum Abbau von Einkommensungleichheiten und damit zur Verteilungsgerechtigkeit beitragen als ein direkter Einkommenstransfer, von dem unklar bleibt, wie viel davon bei den Kindern in Form von besserer Betreuung durch die Familie ankommt. 


\section{Literaturverzeichnis}

Abowd J. M. und H. S. Farber (1982), Job Queues and the Union Status of Workers, Industrial and Labor Relations Review 35 (3), 354-367.

Beblo, M (2001), Die Freizeitlücke zwischen erwerbstätigen Müttern und Vätern - Ein ökonomischer Erklärungsversuch mit Daten der Zeitbudgeterhebung 1991/92, in: Ehling, M., Merz, J. et al. (eds.), Zeitbudget in Deutschland - Erfahrungsberichte der Wissenschaft, Spektrum Bundesstatistik Bd. 17, Stuttgart, 103-116.

Beblo, M. und E. Wolf (2002), The Wage Penalties of Heterogeneous Employment Patterns - An Empirical Analysis for Germany, ZEW Discussion Paper No. 02-45, Mannheim.

Beblo M., D. Beninger und F. Laisney (2004), Family Tax Splitting: A Microsimulation of ist Potential Labour Supply and Intra-household Welfare Effects in Germany, Applied Economics Quarterly 50(3), 231-248.

Beblo M., C. Lauer und K. Wrohlich (2005), Ganztagsschulen und die Erwerbstätigkeit von Müttern. Eine Mikrosimulationsstudie für Deutschland, Zeitschrift für Arbeitsmarktforschung - Journal for Labor Market Research 38(2+3), 357-372.

Blundell R., M. Brewer, P. Haan, A. Shephard (2009), Optimal Income Taxation of Lone Mothers: An Empirical Comparison of the UK and Germany, The Economic Journal 119, F101-F121.

BMFSFJ - Bundesministerium für Familie, Senioren, Frauen und Jugend (2009), Bericht der Bundesregierung, Februar 2009, Berlin.

Biedinger N. und B. Becker (2006), Der Einfluss des Vorschulbesuchs auf die Entwicklung und den langfristigen Bildungserfolg von Kindern - Ein Überblick über internationale Studien im Vorschulbereich, Discussion Paper No. 97, Mannheimer Zentrum für Eu-ropäische Sozialforschung, Mannheim.

Bonin H., W. Kempe und H. Schneider (2003), Household Labor Supply Effects of Low Wage Subsidies in Germany, Schmollers Jahrbuch - Journal of Applied Social Science Studies 123(1), 199-208.

Chevalier A. und T. K. Viitanen (2004), The Supply of Childcare in Britain: Do Mothers Queue for Childcare?, Mimeo.

Clauss, Markus und Stefanie Schubert (2009), Microsimulation Meets General Equilibrium - A New Tool for Applied Policy Analysis, in: Asghar Zaidi, Ann Harding, Paul Williamson (Eds.), New Frontiers in Microsimulation Modelling, Surrey, 557-580.

Creedy J., G. Kalb, H. Kew (2007), Confidence Intervals for Policy Reforms in Behavioural Tax Microsimulation Modelling, Bulletin of Economic Research 59:1, 37-65.

Dearing H., C. Lietz, H. Hofer, R. Winter-Ebmer und K. Wrohlich (2007), Why are mothers working longer hours in Austria than in Germany? A comparative microsimulation study, Fiscal Studies 28(4), 463495.

Fuest C., A. Peichl, C. Bergs und T. Schaefer (2006), Das Familienrealsplitting als Reformoption der Familienbesteuerung, Wirtschaftsdienst 86(10), 639-644.

Hardoy I. und P. Schøne (2008), Incentives to Work: Labour Supply Effects of a Cash-for-Care Subsidy for Non-Western Female Immigrants, Institute for Social Research, Oslo.

Ilmakunnas S. (1997), Public Policy and Child Care Choices. In: Persson I. und C. Jonung (eds.), Economics of the Family and Family Policies, Routledge, London, 178-193.

Kolvenbach F.-J. und D. Taubmann (2006): Neue Statistiken zur Kindertagesbetreuung, Wirtschaft und Statistik 2/2006, 166-171.

Maiterth R. (2004), Verteilungswirkungen alternativer Konzepte zur Familienförderung. Eine empirische Analyse auf Grundlage der Einkommensteuerstatistik des statistischen Bundesamtes, Jahrbücher für Nationalökonomie und Statistik 224(6), 696-730.

McFadden, D. (1974), Conditional logit analysis of qualitative choice behaviour, in: P. Zarembka (Hrsg.), Frontiers in Econometrics, Academic Press, New York, 105-142. 
Naz G. (2004), The impact of cash-benefit reform on parents' labour force participation, Journal of Population Economics 17, 369-383

Opielka M. und M. Winkler (2009), Evaluation der Wirkungen der „Thüringer Familienoffensive“ - Endbericht, Thüringer Ministerium für Soziales, Familie und Gesundheit, und Thüringer Kultusministerium, Erfurt.

Poirier D. J. (1980), Partial Observability in Bivariate Probit Models, Journal of Econometrics 12, $209-217$.

Pfeiffer, Friedhelm und Karsten Reuß (2008), Ungleichheit und die differentiellen Erträge frühkindlicher Bildungsinvestitionen im Lebenszyklus, ZEW Discussion Paper No. 08-001, Mannheim.

Schøne P. (2004), Labour supply effects of a cash-for-care subsidy, Journal of Population Economics 17, 703727

Sipilä J. und J. Korpinen (1998), Cash versus Child Care Services in Finland, Social Policy and Administration 32(3), 263-277.

Spieß C.K., F. Büchel und G.G. Wagner (2003), Children’s School Placement in Germany: Does Kindergarten Attendance Matter?, Early Child Research Quarterly 18, 255-270.

Spieß C.K. und K. Wrohlich (2008), The Parental Leave Benefit Reform in Germany: Costs and Labor Market Oucomes of Moving towards the Nordic Model, Population Research and Policy Review 27, 575-591.

Statistics Norway (2003), Barnehager 2003 - Kindergartens 2003, Oslo.

Statistics Norway (2007), Children in Kindergartens, by age and county, Oslo.

Steiner V. und K. Wrohlich (2004), Household Taxation, Income Splitting and Labor Supply Incentives - A Microsimulation Study for Germany, CESifo Economic Studies 50(3), 541-568.

Steiner V. und Wrohlich K (2008), Introducing Family Tax Splitting in Germany: How Would It Affect the Income Distribution and Work Incentives?, FinanzArchiv 64 (1), 115-142.

van Soest A. (1995), Structural Models of Family Labor Supply: A Discrete Choice Approach, Journal of Human Resources 30, 63-88.

Wagner G.G., J.R. Frick und J. Schupp (2006), Enhancing the Power of Household Panel Studies - The Case of the German Socio-Economic Panel Study (SOEP), Data Documentation 13, DIW, Berlin.

Wrohlich K. (2007), Evaluating Family Policy Reforms Using Behavioural Microsimulation. The Example of Childcare and Income Tax Reforms in Germany, Dissertationsschrift, Freie Universität Berlin. Im Internet veröffentlicht unter http://www.diss.fu-berlin.de/2007/531.

Ziefle A. (2004), Die individuellen Kosten des Erziehungsurlaubs: Eine empirische Analyse der kurz- und längerfristigen Folgen für den Karriereverlauf von Frauen, Zeitschrift für Soziologie und Sozialpsychologie 56, 213-231. 


\section{Tabellen}

Tabelle 1: Simulierte Erwerbsbeteiligung bei Müttern mit Kindern zwischen 13 und 36 Monaten (in \%)

\begin{tabular}{l|cccc}
\hline \hline & Status quo & Betreuungsgeld & $\begin{array}{c}\text { Ausbau der Kin- } \\
\text { derbetreuung }\end{array}$ & Gesamteffekt \\
\hline Nicht-Partizipation & 71,8 & 80,1 & 68,2 & 76,5 \\
Teilzeit & 14,7 & 7,5 & 14,2 & 6,8 \\
Vollzeit & 13,5 & 12,4 & 17,6 & 16,7 \\
\hline \hline
\end{tabular}

Quelle: Simulationsergebnisse auf Basis des SOEP 2002-2006.

Tabelle 2: Simulierte Arbeitsangebotseffekte nach Bevölkerungsgruppe (in \%)

\begin{tabular}{l|cccc}
\hline \hline & \multicolumn{4}{c}{ Veränderungen je 100 Frauen } \\
\cline { 2 - 5 } & Keine Änderung & $\begin{array}{c}\text { Nicht-Partizipation } \\
\text { Partizipation }\end{array}$ & $\begin{array}{c}\text { Partizipation } \\
\text { Nicht-Partizipation }\end{array}$ & $\begin{array}{c}\text { Teilzeit } \\
\text { Vollzeit }\end{array}$ \\
\hline Gesamt & 86,8 & 3,4 & 8,1 & 1,7 \\
Deutsche & 85,4 & 3,1 & 9,1 & 2,4 \\
Ausländer & 91,4 & 3,5 & 4,9 & 0,2 \\
Zweiverdiener & 69,7 & 0,0 & 25,1 & 5,2 \\
Alleinverdiener & 94,1 & 5,9 & 0,0 & 0,0 \\
Hohe Bildung & 93,8 & 2,4 & 2,5 & 1,3 \\
Niedrige Bildung & 84,3 & 3,8 & 9,4 & 2,5 \\
1. Quartil & 88,2 & 7,1 & 4,7 & 0,0 \\
2. Quartil & 77,4 & 3,9 & 15,8 & 2,9 \\
3. Quartil & 86,7 & 1,9 & 8,8 & 2,6 \\
4. Quartil & 94,9 & 0,7 & 3,1 & 1,3 \\
\hline \hline
\end{tabular}

Szenario: Einführung eines Betreuungsgeldes und Ausbau der Kinderbetreuung mit Beseitigung von Rationierungen.

Quelle: Simulationsergebnisse auf Basis des SOEP 2002-2006. 
Tabelle 3: Simulierte Inanspruchnahme von externer Kinderbetreuung bei Müttern mit Kindern zwischen 13 und 36 Monaten (in \%)

\begin{tabular}{l|cccc}
\hline \hline & Status quo & Betreuungsgeld & $\begin{array}{c}\text { Ausbau der Kin- } \\
\text { derbetreuung }\end{array}$ & Gesamteffekt \\
\hline Keine & 61,3 & 69,5 & 56,8 & 65,4 \\
Halbtags & 26,2 & 19,1 & 27,1 & 19,6 \\
Ganztags & 12,5 & 11,4 & 16,1 & 15,0 \\
\hline \hline
\end{tabular}

Quelle: Simulationsergebnisse auf Basis des SOEP 2002-2006.

Tabelle 4: Simulierte Wirkungen auf die Inanspruchnahme externer Kindertagesbetreuung nach Bevölkerungsgruppe (in \%)

\begin{tabular}{|c|c|c|c|c|}
\hline & \multicolumn{4}{|c|}{ Veränderungen je 100 Frauen } \\
\hline & Keine Änderung & $\begin{array}{l}\stackrel{\text { Betreuung }}{\rightarrow} \\
\text { Keine Betreuung }\end{array}$ & $\begin{array}{c}\text { Keine Betreuung } \\
\vec{\rightarrow} \\
\text { Betreuung }\end{array}$ & $\begin{array}{c}\text { Betreuung } \\
\text { halbtags } \\
\rightarrow \\
\text { Betreuung } \\
\text { ganztags }\end{array}$ \\
\hline Gesamt & 86,9 & 8,1 & 4,0 & 1,0 \\
\hline Deutsche & 84,6 & 10,2 & 3,9 & 1,3 \\
\hline Ausländer & 92,0 & 2,9 & 4,9 & 0,2 \\
\hline Zweiverdiener & 73,5 & 20,8 & 4,5 & 1,2 \\
\hline Alleinverdiener & 93,9 & 5,3 & 0,8 & 0,0 \\
\hline Hohe Bildung & 94,3 & 2,6 & 2,3 & 0,8 \\
\hline Niedrige Bildung & 83,8 & 9,5 & 5,6 & 1,1 \\
\hline 1. Quartil & 89,2 & 7,0 & 3,8 & 0,0 \\
\hline 2. Quartil & 77,4 & 15,7 & 5,1 & 1,8 \\
\hline 3. Quartil & 89,5 & 7,6 & 3,2 & 1,7 \\
\hline 4. Quartil & 91,5 & 1,7 & 6,3 & 0,5 \\
\hline
\end{tabular}

Szenario: Einführung eines Betreuungsgeldes und Ausbau der Kinderbetreuung mit Beseitigung von Rationierungen.

Quelle: Simulationsergebnisse auf Basis des SOEP 2002-2006. 
Tabelle 5: Simulierte Einkommenswirkungen nach Bevölkerungsgruppe (Differenz gegenüber Status quo in Euro)

\begin{tabular}{|c|c|c|c|}
\hline & \multicolumn{3}{|c|}{ Status der Mutter im Status quo } \\
\hline & Nicht erwerbstätig & Erwerbstätig & Gesamt \\
\hline Gesamt & 166,9 & 0,7 & 117,9 \\
\hline \multicolumn{4}{|l|}{ davon: } \\
\hline Betreuungsgeld & 148,7 & 36,9 & 115,2 \\
\hline Aufhebung der Rationierung & 1,6 & 20,1 & 7,2 \\
\hline Anpassung der Arbeitszeit & 18,3 & $-64,8$ & $-6,6$ \\
\hline Anpassung der Kinderbetreuung & $-1,7$ & 8,5 & 0,2 \\
\hline Deutsche & 170,8 & $-2,2$ & 115,4 \\
\hline Ausländer & 153,8 & 12,3 & 124,4 \\
\hline Zweiverdiener & - & $-0,4$ & $-0,4$ \\
\hline Alleinverdiener & 166,7 & - & 166,7 \\
\hline Hohe Bildung & 168,4 & 8,9 & 115,2 \\
\hline Niedrige Bildung & 160,9 & $-1,4$ & 128,1 \\
\hline 1. Quartil & 156,2 & 9,7 & 126,6 \\
\hline 2. Quartil & 168,4 & $-0,1$ & 123,8 \\
\hline 3. Quartil & 176,6 & 0,5 & 122,2 \\
\hline 4. Quartil & 166,9 & 4,0 & 99,1 \\
\hline
\end{tabular}

Szenario: Einführung eines Betreuungsgeldes und Ausbau der Kinderbetreuung mit Beseitigung von Rationierungen.

Quelle: Simulationsergebnisse auf Basis des SOEP 2002-2006. 